\title{
MPC-Relevant Prediction-Error Identification
}

\author{
Jørgensen, John Bagterp; Jørgensen, Sten Bay
}

Published in:

American Control Conference 2007

Link to article, DOI:

10.1109/ACC. 2007.4282550

Publication date:

2007

Document Version

Publisher's PDF, also known as Version of record

Link back to DTU Orbit

Citation (APA):

Jørgensen, J. B., \& Jørgensen, S. B. (2007). MPC-Relevant Prediction-Error Identification. In American Control Conference 2007 IEEE. https://doi.org/10.1109/ACC.2007.4282550

\section{General rights}

Copyright and moral rights for the publications made accessible in the public portal are retained by the authors and/or other copyright owners and it is a condition of accessing publications that users recognise and abide by the legal requirements associated with these rights.

- Users may download and print one copy of any publication from the public portal for the purpose of private study or research.

- You may not further distribute the material or use it for any profit-making activity or commercial gain

- You may freely distribute the URL identifying the publication in the public portal

If you believe that this document breaches copyright please contact us providing details, and we will remove access to the work immediately and investigate your claim 


\section{MPC-Relevant Prediction-Error Identification}

\author{
John Bagterp Jørgensen* \\ Informatics and Mathematical Modelling \\ Technical University of Denmark \\ DK-2800 Kgs. Lyngby, Denmark \\ jbj@imm.dtu.dk
}

\author{
Sten Bay Jørgensen \\ Department of Chemical Engineering \\ Technical University of Denmark \\ DK-2800 Kgs. Lyngby, Denmark \\ sbj@kt.dtu.dk
}

\begin{abstract}
A Prediction-error-method tailored for model based predictive control is presented. The prediction-error method studied are based on predictions using the Kalman filter and Kalman predictors for a linear discrete-time stochastic state space model. The linear discrete-time stochastic state space model is realized from a continuous-discrete-time linear stochastic system specified using transfer functions with timedelays. It is argued that the prediction-error criterion should be selected such that it is compatible with the objective function of the predictive controller in which the model is to be applied. The suitability of the proposed prediction error-method for predictive control is demonstrated for dual composition control of a simulated binary distillation column.
\end{abstract}

\section{INTRODUCTION}

Predictive control computes the controls based on a prediction of the outputs. The predictions are based on a model of the system. Original model predictive control algorithms developed for the process industries such as Dynamic Matrix Control [1] and Model Predictive Heuristic Control [2] are based on step an impulse response models for the output predictions and introduce feedback by updating a bias term representing an output disturbance. In contrast to the approach applied by the process industries, the academic system identification community developed predictive controllers based on ARMAX (ARIMAX, CARIMA) models [3]-[5]. Generalized Predictive Control [4] is the most famous implementation of this class of predictive controllers that use an ARMAX model for the filter in generating the feedback as well as for the output predictions. The success of these model based control algorithms and their applications is to a large extent due to existence of efficient methods for generation of the models needed by these controllers.

Modern predictive control is discussed in terms of state space models [6]-[8]. These algorithms are based on a discrete-time linear stochastic state space model. They apply a Kalman filter to compute the current state based on the measurements, and a Kalman predictor for predicting the outputs [9]. State space based model predictive controllers may be derived by realization of input-output models (i.e. FIR, ARX, ARMAX models) in state space form. Subspace identification may be regarded as state space realization of ARX models. However, no advice has been given regarding direct construction of a linear state space model suitable for predictive control. Therefore, better identification methods

\footnotetext{
*Corresponding author
}

for state space based predictive control are requested [10][14].

This paper addresses construction of stochastic linear state space models using the prediction-error-method and a continuous-discrete time linear stochastic transfer function parametrization [15]-[17]. In particular, we argue that for predictive control the parameters in the model should be based on the multi-step prediction error compatible with the predictive controller in which the predictor is to be used. Shah and coworkers [18]-[20] apply a similar multistep approach based on impulse response models and a least-squares criterion. The approach presented in this paper distinguishes itself by being general for linear systems, by applying a maximum likelihood criteria for the prediction errors in the estimator, and in particular by providing models that are tuned for state space model based predictive control in its modern implementation.

\section{Predictive Control}

The predictive controller considered assumes that the system, $\mathcal{S}$, can be described by a linear stochastic discretetime difference equation of the form

$$
\begin{aligned}
\boldsymbol{x}_{k+1} & =A(\theta) \boldsymbol{x}_{k}+B(\theta) u_{k}+\boldsymbol{w}_{k} \\
\boldsymbol{y}_{k} & =C(\theta) \boldsymbol{x}_{k}+\boldsymbol{v}_{k}
\end{aligned}
$$

in which

$$
\left[\begin{array}{c}
\boldsymbol{w}_{k} \\
\boldsymbol{v}_{k}
\end{array}\right] \sim N_{i i d}\left(\left[\begin{array}{l}
0 \\
0
\end{array}\right],\left[\begin{array}{ll}
R_{w w}(\theta) & R_{w v}(\theta) \\
R_{w v}(\theta)^{\prime} & R_{v v}(\theta)
\end{array}\right]\right)
$$

and

$$
\boldsymbol{x}_{0} \sim N\left(\hat{x}_{0 \mid-1}(\theta), P_{0 \mid-1}(\theta)\right)
$$

The system matrices, $(A=A(\theta), B=B(\theta), C=C(\theta))$, are parameterized in terms of the parameter vector, $\theta$. This parameter vector is also used to specify the distribution of the exogenous stochastic variables, i.e. to specify the covariance matrices, $\left(R_{w w}=R_{w w}(\theta), R_{w v}=R_{w v}(\theta), R_{v v}=R_{v v}(\theta)\right)$, of the noise terms as well as to specify the initial condition, $\hat{x}_{0 \mid-1}=\hat{x}_{0 \mid-1}(\theta)$, and its covariance $P_{0 \mid-1}=P_{0 \mid-1}(\theta)$. The states, $\boldsymbol{x}_{k}$, the process noise, $\boldsymbol{w}_{k}$, the measurement noise, $\boldsymbol{v}_{k}$, and the outputs, $\boldsymbol{y}_{k}$, are stochastic vectors. As $\boldsymbol{x}_{0}, \boldsymbol{w}_{k}$, and $\boldsymbol{v}_{k}$ are normally distributed and the system is linear, the states, $\boldsymbol{x}_{k}$, and the outputs, $\boldsymbol{y}_{k}$ are also normally distributed. The inputs, $u_{k}$, are assumed to be deterministic and in particular assumed to be uncorrelated with the process 
measurements, $\boldsymbol{y}_{k}$. This assumption implies that the IO-data, $\left\{y_{k}, u_{k}\right\}_{k=0}^{N-1}$, are collected for a system that operates in open-loop.

Assume that the system matrices, noise covariance matrices, and distribution of the model, $\mathcal{M}$, and the true system, $\mathcal{S}$, are identical. Then prediction uncertainties and errors are due to the stochastic nature of the initial state, the process noise, and the measurement noise, but not due to any systematic trend as a consequence of an incorrect model. In this case, the optimal filter and predictor is the Kalman filter and predictor [9]. Under the same assumptions the separation principle is valid, and the optimal controller for the system can be split into an estimator and a certainty equivalence regulator.Obviously, the true system and the model are never identical. The separation principle does not hold either, as the system is constrained. Nevertheless, predictive control uses the Kalman filter feedback, the Kalman predictor for the output predictions, and separates the controller into an estimator and a regulator. To guarantee offset free control in the case of model-plant mismatch as well as unknown disturbances, the model must be augmented with integrators [21], [22]. For ARMAX models this is achieved by differencing the inputs and outputs [4]. In the face of these approximations and deliberate model modifications introduced to obtain steady-state offset-free control, it is clear that the structure of the estimated model, $\mathcal{M}$, in general will be different from the structure of true system, $\mathcal{S}$. Therefore, it seems most reasonable to view the parameter-estimation purpose to obtain good predictors for the predictive controller rather than accurate parameters in the true unknown model [16].

\section{A. Filter and Predictor}

The filter and predictor used in the predictive controller for the system (1) is the Kalman filter and predictor. The recursions defining the Kalman filter and predictor along with their covariances are stated in this subsection. The filter and predictors are the conditional states, $\boldsymbol{x}_{k+j} \mid \mathcal{I}_{k}$, and the conditional outputs, $\boldsymbol{y}_{k+j} \mid \mathcal{I}_{k}$, given the information vector $\mathcal{I}_{k}$ defined recursively as $\mathcal{I}_{k}=\left\{\mathcal{I}_{k-1}, y_{k}, u_{k-1}\right\}, \mathcal{I}_{0}=\left\{y_{0}\right\}$, and $\mathcal{I}_{-1}=\emptyset$. As all states, $\boldsymbol{x}_{k}$, and outputs, $\boldsymbol{y}_{k}$, are normally distributed, the conditional states, $\boldsymbol{x}_{k+j} \mid \mathcal{I}_{k}$, and the conditional outputs, $\boldsymbol{y}_{k+j} \mid \mathcal{I}_{k}$, are also normally distributed. Normally distributed stochastic variables are completely characterized by their mean and covariance.

The Kalman filter and predictor algorithm stated next is the measurement-time updated Kalman filter and predictor [9]. As new information, $y_{k}$, becomes available, the gains, $K_{f x, k}$ and $K_{f w, k}$, and one-step prediction error, $e_{k}$, of the filter are updated according to

$$
\begin{aligned}
\hat{y}_{k \mid k-1} & =C \hat{x}_{k \mid k-1} \\
e_{k} & =y_{k}-\hat{y}_{k \mid k-1} \\
R_{e, k} & =C P_{k \mid k-1} C^{\prime}+R_{v v} \\
K_{f x, k} & =P_{k \mid k-1} C^{\prime} R_{e, k}^{-1} \\
K_{f w, k} & =R_{w v} R_{e, k}^{-1}
\end{aligned}
$$

The filtered state and filtered process disturbance are normally distributed, i.e. $\boldsymbol{x}_{k} \mid \mathcal{I}_{k} \sim N\left(\hat{x}_{k \mid k}, P_{k \mid k}\right)$ and $\boldsymbol{w}_{k} \mid \mathcal{I}_{k} \sim$ $N\left(\hat{w}_{k \mid k}, Q_{k \mid k}\right)$. The expressions for the filtered conditional means are

$$
\begin{aligned}
& \hat{x}_{k \mid k}=\hat{x}_{k \mid k-1}+K_{f x, k} e_{k} \\
& \hat{w}_{k \mid k}=K_{f w, k} e_{k}
\end{aligned}
$$

and the expressions for the filtered conditional covariances are

$$
\begin{aligned}
P_{k \mid k} & =P_{k \mid k-1}-K_{f x, k} R_{e, k} K_{f x, k}^{\prime} \\
Q_{k \mid k} & =R_{w w}-K_{f w, k} R_{e, k} K_{f w, k}^{\prime}
\end{aligned}
$$

The one-step-ahead prediction of the state, $\boldsymbol{x}_{k+1} \mid \mathcal{I}_{k} \sim$ $N\left(\hat{x}_{k+1 \mid k}, P_{k+1 \mid k}\right)$, and the measured output, $\boldsymbol{y}_{k+1} \mid \mathcal{I}_{k} \sim$ $N\left(\hat{y}_{k+1 \mid k}, R_{k+1 \mid k}\right)$, are also normally distributed. The onestep-ahead prediction conditional means are

$$
\begin{aligned}
\hat{x}_{k+1 \mid k} & =A \hat{x}_{k \mid k}+B \hat{u}_{k \mid k}+\hat{w}_{k \mid k} \\
\hat{y}_{k+1 \mid k} & =C \hat{x}_{k+1 \mid k}
\end{aligned}
$$

and the associated covariances are computed by

$$
\begin{aligned}
& P_{k+1 \mid k}=A P_{k \mid k} A^{\prime}+Q_{k \mid k}-A K_{f x, k} R_{w v}^{\prime}-R_{w v} K_{f x, k}^{\prime} A^{\prime} \\
& R_{k+1 \mid k}=C P_{k+1 \mid k} C^{\prime}+R_{v v}
\end{aligned}
$$

Similarly, the $j$-step-ahead predictions $(j>1)$ of the states, $\boldsymbol{x}_{k+j} \mid \mathcal{I}_{k} \sim N\left(\hat{x}_{k+j \mid k}, P_{k+j \mid k}\right)$, and the outputs, $\boldsymbol{y}_{k+j} \mid \mathcal{I}_{k} \sim$ $N\left(\hat{x}_{k+j \mid k}\right)$, are normally distributed. The $j$-step-ahead prediction conditional means are

$$
\begin{aligned}
\hat{x}_{k+j \mid k} & =A \hat{x}_{k+j-1 \mid k}+B \hat{u}_{k+j-1 \mid k} \\
\hat{y}_{k+j \mid k} & =C \hat{x}_{k+j \mid k}
\end{aligned}
$$

and the associated conditional covariances are computed by

$$
\begin{aligned}
& P_{k+j \mid k}=A P_{k+j-1 \mid k} A^{\prime}+R_{w w} \\
& R_{k+j \mid k}=C P_{k+j \mid k} C^{\prime}+R_{v v}
\end{aligned}
$$

The recursions (2)-(8) specifies the Kalman filter and predictor equations used by the predictive controller completely. Often the $j$-step-ahead $(j>1)$ conditional covariance equations (8) are not used directly in the predictive controller. Equations (2) and (3) are used in the estimator part for forming the filtered states and filtered process disturbances. Equations (4), (5a), and (6a) are used in the estimator part for updating the Kalman filter.

The described Kalman filter and predictor is implemented numerically robust using the array algorithm [9].

\section{B. Regulator}

Given the conditional mean of the filtered state, $\hat{x}_{k \mid k}$, and the conditional mean of the filtered process disturbance, $\hat{w}_{k \mid k}$, the certainty equivalence predictive regulator applies 
equations (5) and (7) for predicting the mean of the conditional outputs, $\hat{y}_{k+j \mid k}$, in the regulator objective function

$$
\begin{aligned}
\phi_{k}= & \frac{1}{2} \sum_{j=1}^{N_{p}}\left(\hat{y}_{k+j \mid k}-r_{k+j \mid k}\right)^{\prime} Q\left(\hat{y}_{k+j \mid k}-r_{k+j \mid k}\right) \\
& +\frac{1}{2} \sum_{j=0}^{N_{c}} \Delta \hat{u}_{k+j \mid k}^{\prime} S \Delta \hat{u}_{k+j \mid k}
\end{aligned}
$$

The objective, $\phi_{k}$, has a finite prediction horizon, $N_{p}$, and computes the optimal predicted inputs, $\left\{\hat{u}_{k+j \mid k}\right\}_{j=0}^{N_{p}-1}=$ $\left\{\hat{u}_{k+j \mid k}\right\}_{j=0}^{N_{c}} \cup\left\{\hat{u}_{k+j \mid k}\right\}_{j=N_{c}+1}^{N_{p}-1}$ by solving

$$
\begin{array}{ll}
\min _{\left\{\hat{u}_{k+j \mid k}\right\}_{j=0}^{N_{p}-1}} & \phi_{k} \\
\text { s.t. } & (5),(7) \\
& \hat{u}_{k+j \mid k} \in \mathbb{U}\left(\hat{x}_{k \mid k}, \hat{w}_{k \mid k}\right) \\
& \hat{y}_{k+j \mid k} \in \mathbb{Y}\left(\hat{x}_{k \mid k}, \hat{w}_{k \mid k}\right)
\end{array}
$$

and using some parametrization of the tail inputs, $\left\{\hat{u}_{k+j \mid k}\right\}_{j=N_{c}+1}^{N_{p}-1}$ [6], [8], [23]-[25]. The sets $\mathbb{U}(\cdot, \cdot)$ and $\mathbb{Y}(\cdot, \cdot)$ denote input constraints, rate of movement input constraints, and output constraints. (10) is a quadratic program with special structure for which efficient solution algorithms exist [26]. Predictive control is implemented in a moving horizon manner, which means that the first optimal control, $\hat{u}_{k \mid k}$, of the optimal control sequence obtained by solving (10), $\left\{\hat{u}_{k+j \mid k}\right\}_{j=0}^{N_{p}-1}$, is implemented on the process, i.e. $u_{k}=\hat{u}_{k \mid k}$. In some implementations, the setup is modified slightly to accommodate the duration of computation and the implemented process input is $u_{k}=\hat{u}_{k \mid k-1}$.

The implemented process input, $u_{k}=\hat{u}_{k \mid k}$, is an implicit function of the output predictions. The objective function in the regulator of the model predictive controller requires multi-step output prediction, i.e. $\hat{y}_{k+j \mid k}$ for $j=1,2, \ldots, N_{p}$. Hence, intuitively it seems natural to estimate the model parameters based on multi-step prediction capabilities compatible with the regulator objective [18]-[20], [27], [28]. This is in contrast to the usual approach in which the parameters are determined based on their single-step onestep-ahead prediction capabilities. In yet another alternative, $N_{p}$ different models are identified, i.e. one model for each single-step $\mathrm{j}$-step-ahead output prediction, $\hat{y}_{k+j \mid k}$ for $j=1,2, \ldots, N_{p}$. This implies that instead of using $\hat{y}_{k+j \mid k}(\theta)$ for $j=1,2, \ldots, N_{p}$, the predictors $\hat{y}_{k+j \mid k}\left(\theta_{j}\right)$ for $j=1,2, \ldots, N_{p}$ are proposed. This multi-model approach has been applied for ARX models [29], [30] and is also adopted in the MUSMAR (multi-step multi-variable adaptive regulator) algorithm for predictive control [31], [32].

\section{Parametrization and Realization}

A parsimonious parametrization of the state space model (1) is obtained using a multivariate stochastic transfer function specification

$$
\begin{aligned}
\boldsymbol{Z}(s) & =G(s ; \theta) U(s)+H(s ; \theta) \boldsymbol{E}(s) \\
\boldsymbol{y}\left(t_{k}\right) & =\boldsymbol{z}\left(t_{k}\right)+\boldsymbol{v}\left(t_{k}\right)
\end{aligned}
$$

in which $U(s)$ is the process input vector, $\boldsymbol{E}(s)$ is a vector with white noise components, $\boldsymbol{Z}(s)$ is the process output vector. $\boldsymbol{v}\left(t_{k}\right)$ is the measurement noise vector and $\boldsymbol{y}\left(t_{k}\right)$ is the measured process output vector at time $t_{k}$. The elements, $\left\{g_{i j}(s)\right\}$ and $\left\{h_{i j}(s)\right\}$, of the transfer function matrices, $G(s)$ and $H(s)$, are rational transfer functions with time delays

$$
\begin{aligned}
g_{i j}(s) & =\frac{b_{i j}(s ; \theta)}{a_{i j}(s ; \theta)} \exp \left(-\tau_{i j}(\theta) s\right) \\
h_{i j}(s) & =\frac{d_{i j}(s ; \theta)}{c_{i j}(s ; \theta)} \exp \left(-\lambda_{i j}(\theta) s\right)
\end{aligned}
$$

(11) is converted to a linear system of delayed stochastic differential equations using an observer-canonical realization. This system is converted to a stochastic discrete-time state space model using a zero-order-hold input representation and the theory for linear stochastic differential equations. Finally, a minimal stochastic discrete-time state space model (1) is obtained by model reduction using the Hankel-norm.

This realization process is conducted numerically by computation of the discrete-time Markov parameters for each SISO system. The resulting minimal discrete-time state space system is realized from the Hankel matrix with all Markov parameters.

\section{Maximum Likelihood Prediction-Error Identification}

The $N_{p}$ multi-step maximum-likelihood multi-step prediction error estimate is compatible with the model predictive controller with a prediction horizon of $N_{p}$. This multi-step maximum likelihood prediction error estimate is

$$
\hat{\theta}=\arg \min _{\theta \in \Theta} V_{M L}(\theta)
$$

in which the likelihood function is

$$
V_{M L}(\theta)=\frac{n_{y} f}{2} \ln (2 \pi)+\frac{1}{2} \sum_{k=-1}^{N-2} \ln \left(\operatorname{det} R_{k}\right)+\epsilon_{k} R_{k}^{-1} \epsilon_{k}
$$

$f=N_{p}\left[N-\frac{1}{2}\left(N_{p}-1\right)\right], \boldsymbol{\epsilon}_{k}=\boldsymbol{Y}_{k}-\hat{Y}_{k}(\theta), R_{k}=\left\langle\boldsymbol{\epsilon}_{k}, \boldsymbol{\epsilon}_{k}\right\rangle$, and

$$
\boldsymbol{Y}_{k}=\left[\begin{array}{c}
\boldsymbol{y}_{k+1} \\
\boldsymbol{y}_{k+2} \\
\vdots \\
\boldsymbol{y}_{k+N_{p}}
\end{array}\right] \hat{Y}_{k}(\theta)=\left[\begin{array}{c}
\hat{y}_{k+1 \mid k}(\theta) \\
\hat{y}_{k+2 \mid k}(\theta) \\
\vdots \\
\hat{y}_{k+N_{p} \mid k}(\theta)
\end{array}\right]
$$

$\left\{\left(y_{k}, u_{k}\right)\right\}_{k=0}^{N-1}$ is the input-output data for which the estimation is performed. Based on these data, $Y_{k}$ denotes the realization of $\boldsymbol{Y}_{k}$ and $\epsilon_{k}=Y_{k}-\hat{Y}_{k}(\theta) . R_{k}$ has a special structure that should be utilized in the efficient solution of this multi-step maximum-likelihood estimation problem.

\section{WOOD AND BERRY Distillation EXAMPLE}

In this section we demonstrate the proposed multi-step prediction-error identification procedure for the Wood and Berry distillation column separating methanol and water [33], [34]. The Wood and Berry distillation column model is

$$
Y(s)=G(s) U(s)+G_{d}(s) D(s)
$$



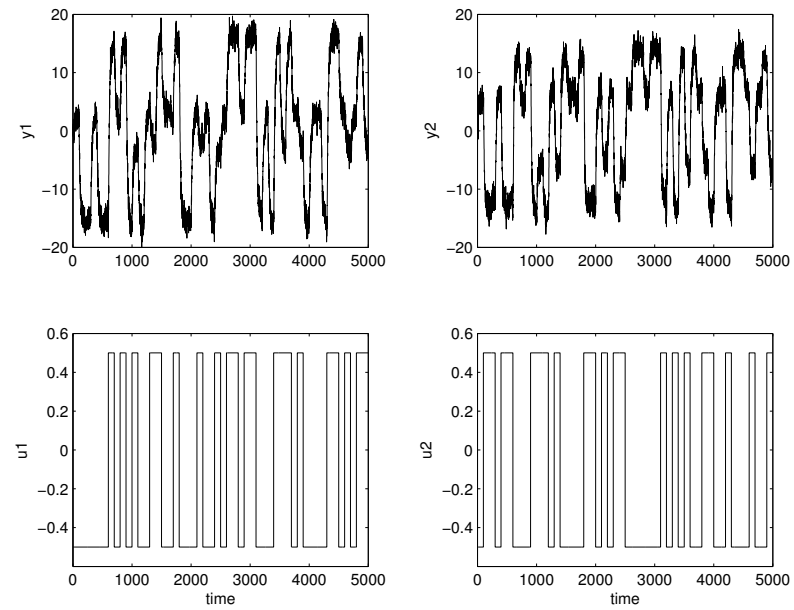

Fig. 1. IO-data for the Wood and Berry distillation column simulated using the model defined by (18) with the transfer functions (17). $\sigma=1$ and $r_{1}=r_{2}=1.0$. The inputs, $\{u(t)\}$, are PRBS with bandwidth [ $\left[\begin{array}{ll}0 & 0.01\end{array}\right]$ and levels $\left[\begin{array}{ll}-0.5 & 0.5\end{array}\right]$.

with

$$
\begin{aligned}
G(s) & =\left[\begin{array}{ll}
\frac{12.8 e^{-s}}{16.7 s+1} & \frac{-18.9 e^{-3 s}}{21.0 s+1} \\
\frac{6.6 e^{-7 s}}{10.9 s+1} & \frac{-19.4 e^{-3 s}}{14.4 s+1}
\end{array}\right] \\
G_{d}(s) & =\left[\begin{array}{l}
\frac{3.8 e^{-8.1 s}}{14.9 s+1} \\
\frac{4.9 e^{-3.4 s}}{13.2 s+1}
\end{array}\right]
\end{aligned}
$$

The variables in the model are: $y_{1}$ is the overhead methanol mole fraction, $y_{2}$ is the bottom product methanol mole fraction, $u_{1}$ is the overhead reflux flow rate, $u_{2}$ is the bottoms steam flow rate, and $d$ is the column feed flow rate.

The output data for the Wood and Berry distillation column is generated using the stochastic model

$$
\begin{aligned}
\boldsymbol{Z}(s) & =G(s) U(s)+G_{d}(s)(D(s)+\sigma \boldsymbol{E}(s)) \\
\boldsymbol{y}\left(t_{k}\right) & =\boldsymbol{z}\left(t_{k}\right)+\boldsymbol{v}\left(t_{k}\right)
\end{aligned}
$$

with $\boldsymbol{E}(s)$ being white noise and $\sigma=1$. The measurement noise is

$$
\boldsymbol{v}\left(t_{k}\right) \sim N_{i i d}\left(\left[\begin{array}{l}
0 \\
0
\end{array}\right],\left[\begin{array}{cc}
r_{1}^{2} & 0 \\
0 & r_{2}^{2}
\end{array}\right]\right)
$$

in which $r_{1}=r_{2}=1.0$. The sampling time of the system is $T_{s}=1.0$. In the identification experiment $u$ is a pseudo random binary sequence and the systematic feed flow rate deviation, $d$, is set to zero.

The IO-data generated for this system and used for identification is plotted in figure 1. It is apparent that outputs are highly co-linear, i.e.the system is ill-conditioned. This is a well known phenomenon for distillation columns and may in many cases require closed-loop identification or specially designed perturbations suitable for the directionality of the plant [35]-[37].

\section{A. Identification of a Control Relevant Model}

In the following the generated open-loop data will be used for estimation of a process model, $\hat{G}(s)$, and a disturbance

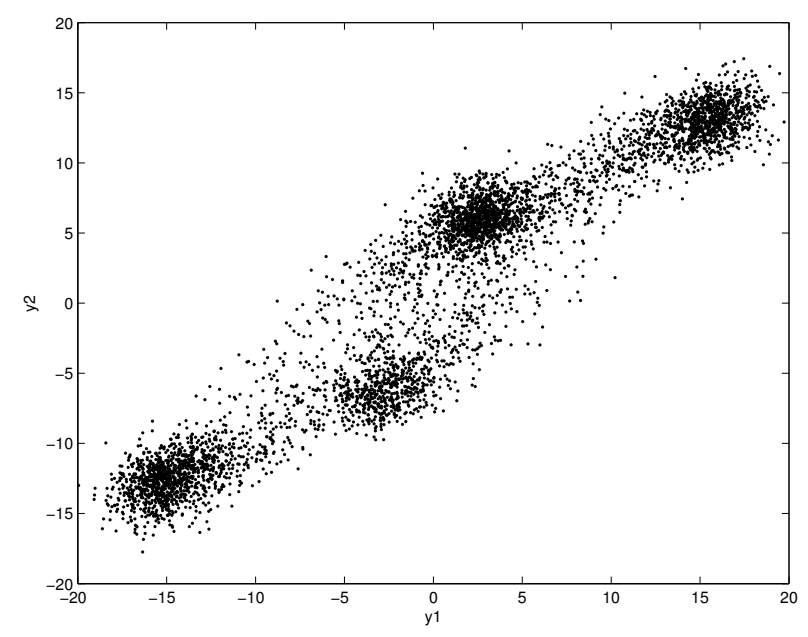

Fig. 2. Phase plane plot of the output data of the Wood and Berry distillation column simulated using the model defined by (18) with the transfer functions (17). $\sigma=1$ and $r_{1}=r_{2}=1.0$. The inputs, $\{u(t)\}$, are PRBS with bandwidth [ $\left[\begin{array}{ll}0 & 0.01\end{array}\right]$ and levels $\left[\begin{array}{ll}-0.5 & 0.5\end{array}\right]$. The figure shows that the output data are highly co-linear.

model, $\hat{H}(s)$. The application of this model for predictive control is demonstrated.

The estimated model is of the form

$$
\begin{aligned}
\hat{\boldsymbol{Z}}(s) & =\hat{G}(s) U(s)+\hat{H}(s) \hat{\boldsymbol{E}}(s) \\
\boldsymbol{y}\left(t_{k}\right) & =\hat{\boldsymbol{z}}\left(t_{k}\right)+\hat{\boldsymbol{v}}\left(t_{k}\right)
\end{aligned}
$$

in which $\hat{G}(s)$ is a transfer function with the same structure as $G(s)$. The disturbance model, $\hat{H}(s)$, has the structure

$$
H(s)=\left[\begin{array}{cc}
h_{11}(s) & 0 \\
0 & h_{22}(s)
\end{array}\right]
$$

with

$$
h_{i i}(s)=\frac{1}{s} \frac{\sigma_{i i}}{\gamma_{i i} s+1} \quad i=1,2
$$

Note that the disturbance model is equipped with integrators in order to ensure steady-state offset-free control when the model is applied in a predictive controller [21], [22]. Using the multi-step maximum likelihood criterion, the estimated model transfer functions are

$$
\begin{aligned}
& \hat{G}(s)=\left[\begin{array}{ll}
\frac{13.21 e^{-0.84 s}}{17.20 s+1} & \frac{-18.52 e^{-3.34 s}}{20.67 s+1} \\
\frac{6.72 e^{-7.69 s}}{10.03 s+1} & \frac{-19.28 e^{-3.07 s}}{14.77 s+1}
\end{array}\right] \\
& \hat{H}(s)=\left[\begin{array}{cc}
\frac{1}{s} \frac{0.18}{0.16 s+1} & 0 \\
0 & \frac{1}{s} \frac{0.27}{0.16 s+1}
\end{array}\right]
\end{aligned}
$$

and the estimated covariance of the measurement noise is

$$
\hat{R}_{v v}=\left[\begin{array}{cc}
1.03^{2} & 0 \\
0 & 1.04^{2}
\end{array}\right]
$$

In this particular disturbance model, we have not utilized that the impact of the actual disturbance on the outputs are correlated even though this is evident from the estimated disturbance model. This implies that the estimated model is essentially two MISO models.

The step responses of the estimated transfer function, $\hat{G}(s)$, and the true transfer function, $G(s)$, are shown in 

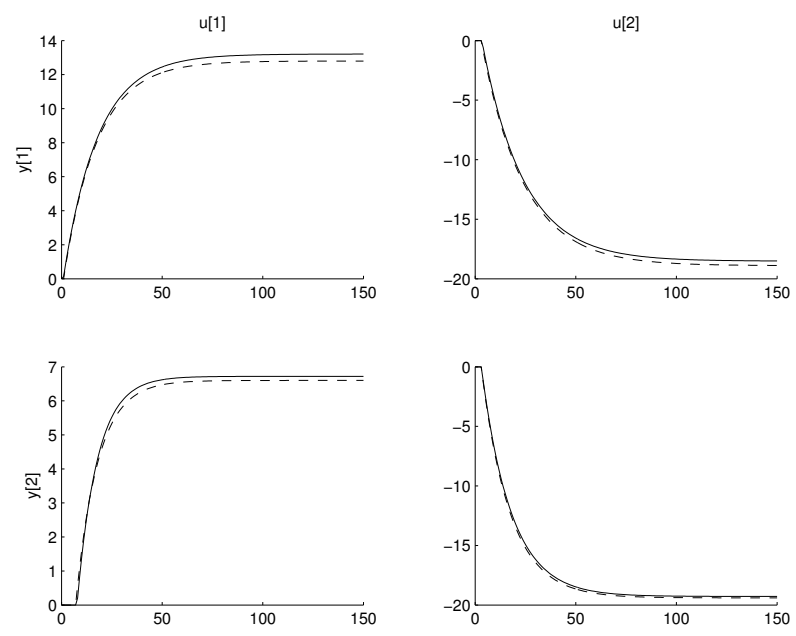

Fig. 3. Step responses for the deterministic part of the Wood and Berry distillation column model. Solid line: Estimated model. Dashed line: True model.

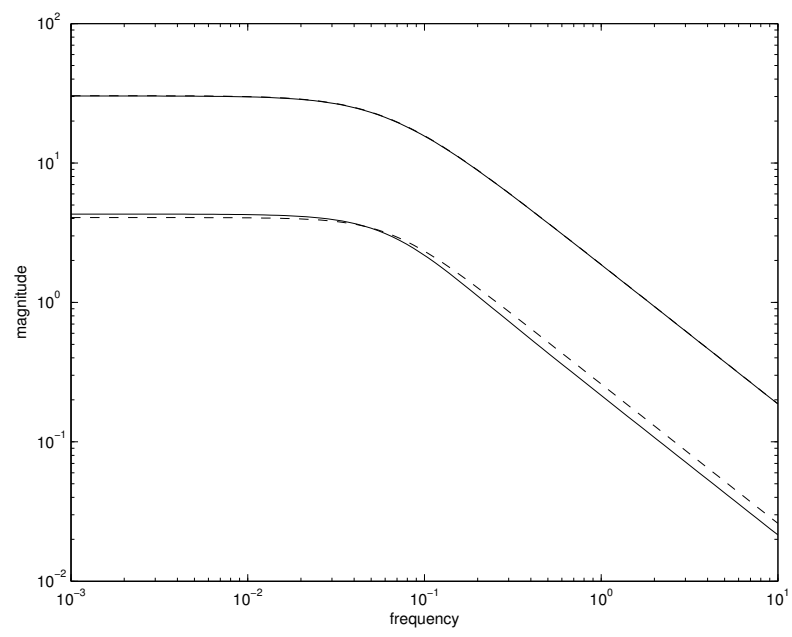

Fig. 4. Low and high gain plots for the true model (dashed line) and the estimated model (solid line) of the Wood and Berry distillation column. It is evident that the error of the estimated model is almost entirely in the low gain direction, while the gain in the high gain direction is estimated accurately.

figure 3. It is evident that the estimated model represents the true model well. To illustrate the identification consequences of having co-linear output data as shown in figure 2, the high gain and low gain direction gains are plotted as function of frequency in figure 4. It is evident that the gain in the high gain direction is estimated accurately, while there is some error in the estimate of the gain in the low gain direction. This phenomena is well known for ill-conditioned plants and can be overcome by closed-loop identification or identification experiments taking the high-gain and low-gain direction into account.

\section{B. Model Predictive Control}

The suitability of the proposed identification method for predictive control is validated by application of the identified
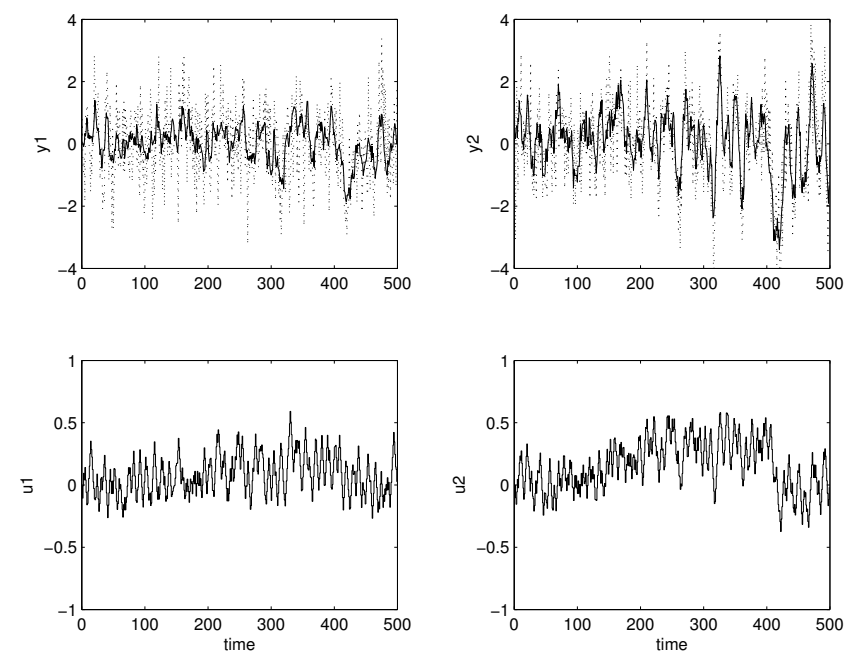

Fig. 5. The Wood and Berry distillation column controlled by a predictive controller. Top: Outputs ( $z$, solid line) and measured outputs ( $y$, dotted line) Bottom: Controlled process inputs, $u$.
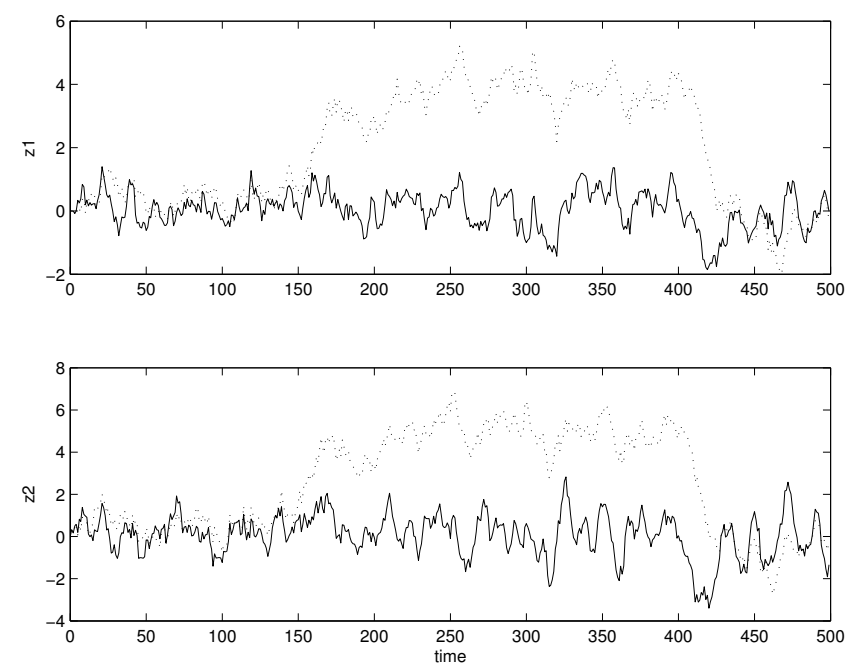

Fig. 6. The process outputs, $z$, for the Wood and Berry distillation column controlled by a predictive controller (solid line) and the identical scenario without control (dotted line).

model (23) for the design of a constrained multivariable predictive controller. This controller is tested in a simulation using (18) as the plant. The transfer functions are defined by (17), $\sigma=1$ and the measurement noise covariance is defined by (19). At time $t=150$ a deterministic feed flow step disturbance, $d=1$, occurs. This disturbance is unknown to the controller. At time $t=400$, the disturbance disappears again.

The performance of the model predictive controller is shown in figure 5. In the upper plots the noise free outputs, $z_{1}$ and $z_{2}$, as well as the measurements, $y_{1}$ and $y_{2}$, are shown. The measurement noise is significant. Its effect on the measured output is of the same order of magnitude as the effect of the process noise. This corresponds to the common industrial plant using low resolution sensors contaminated 
with a high level of measurement noise. In this situation, no control system can completely eliminate the effect of the process noise on the outputs. However, as is evident by figure 6 which compares the open-loop outputs, $\left(z_{1}\right.$ and $z_{2}$, dotted line), to the closed-loop outputs, $\left(z_{1}\right.$ and $z_{2}$, solid line), the controller rejects the disturbance and performs marginally better than the no-control (open-loop) case in the situation with only white process noise. This closedloop performance of the constrained predictive controller indicates that the proposed prediction-error- methodology is suitable and feasible for identification of models that can be used by a predictive controller. For the performance of the controller in the situation with model-plant mismatch as well as unmeasured disturbance, it is important that the noise model is equipped with integrators. In addition identification of parameters in stochastic transfer functions with delays have proven feasible and very useful for specification of predictive controllers.

\section{CONCLUSION}

A constructive method for estimation of parameters in continuous-discrete-time stochastic systems parametrized by transfer functions with time delays has been described. The method applies a multi-step maximum-likelihood predictionerror criterion. The predictions are generated using the Kalman filter and Kalman predictor for a stochastic linear discrete-time state space model, which is a realization of a continuous-discrete-time stochastic transfer function model with time delays. The multi-step prediction-error criteria may be selected such that it is compatible with the optimization criterion in the intended predictive control application. The feasibility of the suggested approach for predictive control is demonstrated using the Wood and Berry [33] distillation example. In particular, the design of predictive controllers using continuous-discrete-time stochastic models specified by transfer functions with delays has proven very convenient.

\section{REFERENCES}

[1] C. R. Cutler and B. L. Ramaker, "Dynamic matrix control - a computer control algorithm," in Joint Automatic Control Conference. ACC, 1980.

[2] J. Richalet, A. Rault, J. L. Testud, and J. Papon, "Model predictive heuristic control: Applications to industrial processes," Automatica, vol. 14, pp. 413-428, 1978.

[3] G. E. P. Box, G. M. Jenkins, and G. C. Reinsel, Time Series Analysis. Forecasting and Control, 3rd ed. New Jersey: Prentice-Hall, 1994.

[4] D. W. Clarke, C. Mohtadi, and P. S. Tuffs, "Generalized predictive control. part 1: The basic algorithm," Automatica, vol. 23, pp. 137148, 1987.

[5] R. R. Bitmead, M. Gevers, and V. Wertz, Adaptive Optimal Control. The Thinking Man's GPC. New York: Prentice-Hall, 1990.

[6] K. R. Muske and J. B. Rawlings, "Model predictive control with linear models," AIChE J., vol. 39, pp. 262-287, 1993.

[7] J. B. Rawlings, "Tutorial overview of model predictive control," IEEE Control Systems Magazine, vol. 20, pp. 38-52, 2000.

[8] J. M. Maciejowski, Predictive Control with Constraints. Harlow, England: Prentice Hall, 2002.

[9] T. Kailath, A. H. Sayed, and B. Hassibi, Linear Estimation. Prentice Hall, 2000.

[10] M. Morari and J. H. Lee, "Model predictive control: Past, present and future," Computers and Chemical Engineering, vol. 23, pp. 667-682, 1999.
[11] S. B. Jørgensen and J. H. Lee, "Recent advances and challenges in process identification," in Chemical Process Control - 6: Assessment and New Directions for Research, J. B. Rawlings and B. A. Ogunnaike, Eds., January 2001.

[12] H. Hjalmarsson, "From experiments to closed loop control," in 13th IFAC Symposium on System Identification, 27-29 August 2003, Rotterdam, The Netherlands. IFAC, 2003.

[13] M. Gevers, "A personal view on the development of system identification," in 13th IFAC Symposium on System Identification, 27-29 August 2003, Rotterdam, The Netherlands. IFAC, 2003.

[14] — " "Identification for control: Achievements and open problems," in DYCOPS 7, Cambridge, Massachussetts USA, July 5-7. IFAC, 2004.

[15] K. J. Åström, "Maximum likelihood and prediction error methods," Automatica, vol. 16, pp. 551-574, 1980.

[16] L. Ljung, System Identification. Theory for the User, 2nd ed. Upper Saddle River, NJ: Prentice Hall, 1999.

[17] N. R. Kristensen, H. Madsen, and S. B. Jørgensen, "Parameter estimation in stochastic grey-box models," Automatica, vol. 40, pp. 225-237, 2004.

[18] D. S. Shook, C. Mohtadi, and S. L. Shah, "A control-relevant identification strategy for GPC," IEEE Transactions on Automatic Control, vol. 37, pp. 975-980, 1992.

[19] R. B. Gopaluni, R. S. Patwardhan, and S. L. Shah, "The nature of data pre-filters in MPC relevant identification - open- and closed-loop issues," Automatica, vol. 39, pp. 1617-1626, 2003.

[20] — "MPC relevant identification - tuning the noise model," Journal of Process Control, vol. 14, pp. 699-714, 2004.

[21] K. R. Muske and T. A. Badgwell, "Disturbance modeling for offsetfree linear model predictive control," Journal of Process Control, vol. 12, pp. 617-632, 2002.

[22] G. Pannocchia and J. B. Rawlings, "Disturbance models for offset-free model-predictive control," AIChE J., vol. 49, pp. 426-437, 2003.

[23] C. E. Garcia, D. M. Prett, and M. Morari, "Model predictive control: Theory and practice - a survey," Automatica, vol. 25, pp. 335-348, 1989.

[24] P. O. M. Scokaert and J. B. Rawlings, "Constrained linear quadratic regulation," IEEE Trans. Automat. Contr., vol. 43, pp. 1163-1169, 1998.

[25] D. Q. Mayne, J. B. Rawlings, C. V. Rao, and P. O. M. Scokaert, "Constrained model predictive control: Stability and optimality," $\mathrm{Au}$ tomatica, vol. 36, pp. 789-814, 2000.

[26] J. B. Jørgensen, J. B. Rawlings, and S. B. Jørgensen, "Numerical methods for large scale moving horizon estimation and control," in DYCOPS 7, Cambridge, MA, July 5-7, 2004.

[27] T. Söderström and P. Stoica, System Identification. New York: Prentice Hall, 1989.

[28] P. Stoica and A. Nehorai, "On multistep prediction error methods for time series models," Journal of Forecasting, vol. 8, pp. 357-368, 1989.

[29] J. A. Rossiter and B. Kouvaritakis, "Modelling and implicit modelling for predictive control," International Journal of Control, vol. 74, pp. 1085-1095, 2001.

[30] R. Haber, U. Schmitz, and R. Bars, "Long-range optimal model and multi-step-ahead prediction identification for predictive control," in 13th IFAC Symposium on Identification, Rotterdam, The Netherlands, 27-29 August 2003.

[31] C. Greco, G. Menga, E. Mosca, and G. Zappa, "Performance improvements of self tuning controllers by multistep horizons: The MUSMAR approach," Automatica, vol. 20, pp. 681-700, 1984.

[32] E. Mosca, Optimal, Predictive, and Adaptive Control. Prentice-Hall, 1995.

[33] R. K. Wood and M. W. Berry, "Terminal composition control of a binary distillation column," Chemical Engineering Science, vol. 29, p. 1808, 1973.

[34] B. A. Ogunnaike and W. H. Ray, Process Dynamics, Modeling, and Control. New York: Oxford University Press, 1994.

[35] H. W. Andersen, M. Kümmel, and S. B. Jørgensen, "Dynamics and identification of a binary distillation column," Chemical Engineering Science, vol. 44, pp. 2571-2581, 1989.

[36] C.-V. Koung and J. F. MacGregor, "Identification for robust multivariable control: The design of experiments," Automatica, vol. 30, pp. 1541-1554, 1994.

[37] Y. Zhu, Multivariable System Identification for Process Control. Amsterdam: Pergamon, 2001. 